\title{
La legislación aduanera colombiana y su influencia en el desarrollo del poder naval
}

\author{
Colombian customs legislation and its influence on the development of naval power
}

\author{
Christian Eliecer González-Miranda ${ }^{1}$ (i)
}

${ }^{1}$ Escuela Superior de Guerra, Colombia.Email: gonzalezc@esdegue.edu.co

Para citar este artículo: Christian, E. M. (2020). La legislación aduanera colombiana y su influencia en el desarrollo del poder naval. Clío América, 14(27), 409-421. http://dx.doi.org/10.21676/23897848.3688

Recibido: 15 noviembre de 2019

Aceptado: 07 de febrero de 2020

Publicado en línea: marzo 03 de 2020

Palabras clave: aranceles; diferimiento arancelario; poder marítimo; poder naval; conciencia marítima.

JEL: O21; H23; H24

\section{Keywords:} tariffs; tariff deferral; maritime power; naval power; maritime consciousness

\section{RESUMEN}

La legislación aduanera colombiana busca orientar y preservar los derechos aduaneros mediante el uso de barreras económicas que tienen como finalidad la protección de la producción nacional y el mantenimiento de sus regulaciones, que facilitan la participación del país en un comercio internacional dinámico. Sin embargo, la política comercial colombiana desconoce las necesidades del sector defensa, especialmente por la inobservancia de conceptos como "poder marítimo", "poder naval" y "conciencia marítima". Por lo tanto, se presentará un análisis del arancel de aduanas vigente, que deja en evidencia una afectación al desarrollo del poder naval, relacionado con las subpartidas arancelarias específicas que son utilizadas en los procesos de mantenimiento de la Fuerza Naval, las cuales limitan la ejecución de proyectos de modernización de las capacidades estratégicas de la Armada República de Colombia (ARC). Por lo anterior, se presentarán las subpartidas arancelarias utilizadas de forma recurrente en dichos proyectos, que son de uso exclusivo para los componentes navales y que generan actualmente pago de aranceles, a pesar de que no afectan la producción nacional. Por último, se planteará una propuesta de modificación basada en diferimientos arancelarios conforme a los vacíos encontrados en la actual legislación aduanera.

\section{ABSTRACT}

Colombian customs legislation seeks guidance and preservation of customs rights using economic barriers that aim to protect national production and maintain its regulations that facilitate the country's participation in dynamic international trade. However, Colombian trade policy ignores the needs of the defense sector, especially due to the innervation of concepts such as "maritime awareness", "maritime power" and "naval power". Therefore, an analysis of the current customs tariff is presented, revealing an impact on the development of the naval power related to the specific tariff subheadings used in the processes of maintenance of the Naval Force, which are limited the execution of projects of modernization of strategic capabilities of the Colombian Republic Navy. Therefore, the tariff subheadings used regularly in these projects are presented, which are for the exclusive use of naval components and which are currently paying tariffs, even though they do not affect domestic production. Lastly, a proposal for modification based on tariff deferrals will be proposed following the gaps found in the current customs legislation. 


\section{LA LEGISLACIÓN ADUANERA COLOMBIANA Y SU INFLUENCIA EN EL DESARROLLO DEL PODER NAVAL}

\section{INTRODUCCIÓN}

Colombia es un país que posee el poder de usar los medios necesarios para cumplir con las funciones definidas en el orden constitucional; sin embargo, quizá en todos los niveles del Estado se debe interiorizar el concepto de "poder", observando la capacidad que posee para satisfacer las necesidades a favor de sus fines e intereses, sobre todo cuando se trata de mantener la integridad territorial como un factor determinante para la supervivencia en la región (Castex, 1939).

Al mismo tiempo, se debe tener claro que por medio del poder marítimo se podrá garantizar el uso del mar en beneficio de los intereses de la nación, por medio del empleo del poder naval suficiente para defender dichos intereses de los enemigos del Estado, bajo una voluntad estratégica marcada por una visión política como factor multiplicador y decisivo en los contextos de conflictos presentes y futuros (Hill, 1990).

Es necesario recalcar que el poder naval, como componente militar del poder marítimo, tiene el propósito de contribuir a la seguridad y a la defensa que faciliten el desarrollo económico y el bienestar de la nación. No obstante, esto demanda el uso de las capacidades estratégicas representadas en la Fuerza Naval, tales como buques, submarinos, aeronaves, entre otros artefactos navales que deben ser mantenidos con el apoyo de una voluntad política firme y realista que valore la citada capacidad (Mahan, 2007).

Por dichas razones, es importante para un Estado velar por el óptimo mantenimiento de su Fuerza Naval y evitar que las trivialidades de las legislaciones internas limiten su desarrollo. Por el contrario, debe persistir la conciencia marítima en todos los nacionales para incentivar el fortalecimiento de las capacidades que soportan el empleo del poder naval (Till, 2007).

En el mismo sentido, se debe analizar el ámbito económico como base para soportar las necesidades que demanda el país para administrar el poder ante sus oponentes y, por ello, es oportuno revisar el vínculo existente desde los inicios de la apertura económica colombiana y la implementación de medidas arancelarias como consecuencia del fenómeno de la globalización (Carrero, 2009).

Todo inicia cuando el Estado se sometió a una carrera por reglamentar la legislación aduanera del país con una visión de competitividad que facilitara el intercambio comercial con otros países. Para esto fue necesario implementar una regulación arancelaria que debía obedecer a las reglas generales emitidas por la Organización Mundial de Comercio - OMC (Ocampo y Villar, 1992).

De aquí que la buena visión del país por ejercer una protección eficaz a los gremios exportadores impulsara la expedición del primer Arancel de Aduanas en Colombia, alineado a las directrices de la OMC, los conceptos para unificar los sistemas de clasificación arancelarias y las definiciones de nomenclaturas para las mercancías (Acuña, 1994).

Con sano criterio, el Estado estableció el Arancel de Aduanas, en 1995, con el objetivo de regular sin distinciones, mantener el equilibrio económico y reducir formalidades aduaneras para generar el escenario propicio de liberalización comercial e implementación de la política comercial por parte del país, como un paso definitivo y de no retorno de la apertura económica iniciada en los 90 (Donadio, 2013).

No obstante, la apertura económica fue un proceso complejo para Colombia que apostó, en esta nueva dinámica, por el crecimiento financiero del país, esperando resultados positivos que permitieran una real participación en el comercio internacional, bajo una nueva tendencia de integración con otros países conforme a los intereses negociados (Steinberg, 2004).

De este modo, Colombia ingresó al sistema multilateral de comercio para aumentar los flujos comerciales en las exportaciones y lograr la disminución real de la dependencia comercial de socios comerciales tradicionales. Además, inició el reto de administrar las medidas de proteccionismo comercial que alteran, positiva o negativamente, el normal desarrollo del comercio internacional (Appleyard, 2003).

En este punto es importante revisar el factor "precio de la mercancía", que influye en el análisis de la demanda u oferta del Gobierno, como consecuencia de la dinámica comercial que no puede evitar la participación del componente arancelario; más aún cuando uno de los intereses vitales de un Estado es el ejercicio del recaudo por gravámenes arancelarios con el fin de brindar protección a los productores nacionales frente a precios externos al país (Rivera, 1993).

Sumado a esto y conforme a su política comercial, 
Colombia buscó impulsar las exportaciones de las mercancías con mayor potencial de rentabilidad en los países con mayor demanda del producto, basándose en un análisis del precio nacional frente al precio internacional (Chacholiades, 1992).

Por lo tanto, el funcionamiento práctico de la dinámica comercial con los aranceles se basa en un costeo que suma el arancel antes de autorizar el ingreso de la mercancía al territorio aduanero nacional, definido como la demarcación que abarca todo el territorio nacional donde aplica la legislación aduanera y actúa el Estado colombiano (Ministerio de Comercio, Industria y Turismo -MINCIT, 2016).

De igual forma, es oportuno evocar el concepto de "derecho de aduana", por la fuerte relación que tiene con la mercancía y los aranceles, definido como la actividad que consiste en gravar un arancel de acuerdo con su nomenclatura arancelaria de la mercancía. Dicha tarifa se determina conforme las características del bien sobre el cual recae el gravamen (Queralt, 2007).

En el mismo sentido, los derechos de aduana representan el conjunto de normas que respaldan el ejercicio de las aduanas como autoridad en los estados, en cumplimiento de su función de control sobre el tráfico de mercancías que ingresan y salen del territorio aduanero nacional, bajo lineamientos y reconocimientos de regímenes especiales que facilitan el desarrollo de las operaciones aduaneras (Basaldúa, 1998).

Debido a la relevancia del tema, el Ministerio de Defensa Nacional - Mindefensa, a través de la ARC para el mantenimiento de la Fuerza Naval, parte integral del poder naval, motivó la conformación de dependencias como la Dirección de Comercio Exterior de la ARC (Dicomex), gestionando el acceso a regímenes especiales descritos por la Dirección de Impuestos y Aduanas Nacionales - DIAN, los cuales permiten ejecutar operaciones de importación, exportación y tránsito aduanero (de aquí en adelante, operaciones aduaneras).

\section{METODOLOGÍA}

Se utiliza un enfoque de tipo reflexivo, basado en un análisis legislativo desde el punto de vista del comercio exterior en Colombia, que abarca desde la apertura económica hasta la actualidad, para luego sumergirse en el caso puntual del Mindefensa; en especial, de la ARC representada por la Dicomex. Luego de la introducción, se podrán identificar los vacíos legislativos que afectan el mantenimiento del poder naval en Colombia y, por ende, del poder marítimo del país, basándose en la revisión de las operaciones aduaneras de Dicomex, del año 2012 al 2019, con el fin de plasmar una tendencia y afectación al mantenimiento del poder naval.

Por último, se analizarán las partidas arancelarias recurrentes, mencionado el período de estudio, para presentar una propuesta de diferimiento arancelario, que puede ser motivada ante MINCIT para modificar el Decreto 2153 del 2017, en pro de los desarrollos futuros de la ARC y, por ende, del poder naval del país.

\section{RESULTADOS}

\section{Inmersión en la Gestión Aduanera de la ARC}

El Mindefensa, a través de la ARC, viene ejecutando los programas de modernización, actualización tecnológica y renovación de las capacidades estratégicas de la Fuerza Naval, que aporta al mantenimiento efectivo del poder naval del país en la última década; esto quedó demostrado en el desarrollo exitoso del programa Plan Orión, en sus fases I y II (ARC, 2015).

Desde 1996, la ARC cuenta con la figura de "depósito habilitado", otorgada mediante resolución DIAN número 6592 de 1996; así también, posee el reconocimiento e inscripción como Usuario Aduanero Permanente (UAP) a través de la Dicomex, bajo la autorización recibida en el 2005 mediante resolución DIAN número 0970 de 2005, teniendo, con esto, la autonomía para realizar trámites ante la Unidad Administrativa Especial de la DIAN (DIAN, 2005).

Los mencionados beneficios, obtenidos por la ARC, han permitido sostener las operaciones aduaneras para el mantenimiento de la Fuerza Naval y, por ende, del poder naval colombiano. Dichas operaciones se efectúan en los predios y en las bodegas de la ARC que fueron registrados ante la DIAN conforme a la figura de depósito habilitado. Cabe resaltar que este tipo de reconocimiento por parte de la DIAN está sujeto a vigencia de cinco años; sin embargo, gracias a la gestión de la Jefatura de Operaciones Logísticas de la ARC se ha podido renovar y la capacidad sigue vigente para la Fuerza Naval (DIAN, 1996).

Los roles adquiridos por la mencionada dependencia de la ARC fueron un acierto estratégico, toda vez que permitieron a la ARC gozar de la autonomía para desarrollar las operaciones aduaneras ante la DIAN, como soporte logístico fundamental para la celeridad de los trámites de nacionalización, disminución de los costos de intermediación aduanera y profundización de los conocimientos por parte del personal orgánico de la 
Dicomex.

Teniendo en cuenta que la Dicomex posee las capacidades de desarrollar con fuerza propia las operaciones aduaneras que demanda el mantenimiento de la Fuerza Naval, a continuación se analizan las operaciones realizadas durante el período 2012-2019, arrojando un total de 2081 operaciones aduaneras (un promedio de 260 operaciones por año) dentro del periodo descrito y que será utilizado a lo largo del presente artículo. Esto se ve reflejado en la Figura 1, en la que se evidencia un nivel máximo de 406 operaciones aduaneras en el 2014 y un mínimo de 153 operaciones aduaneras durante el 2019.

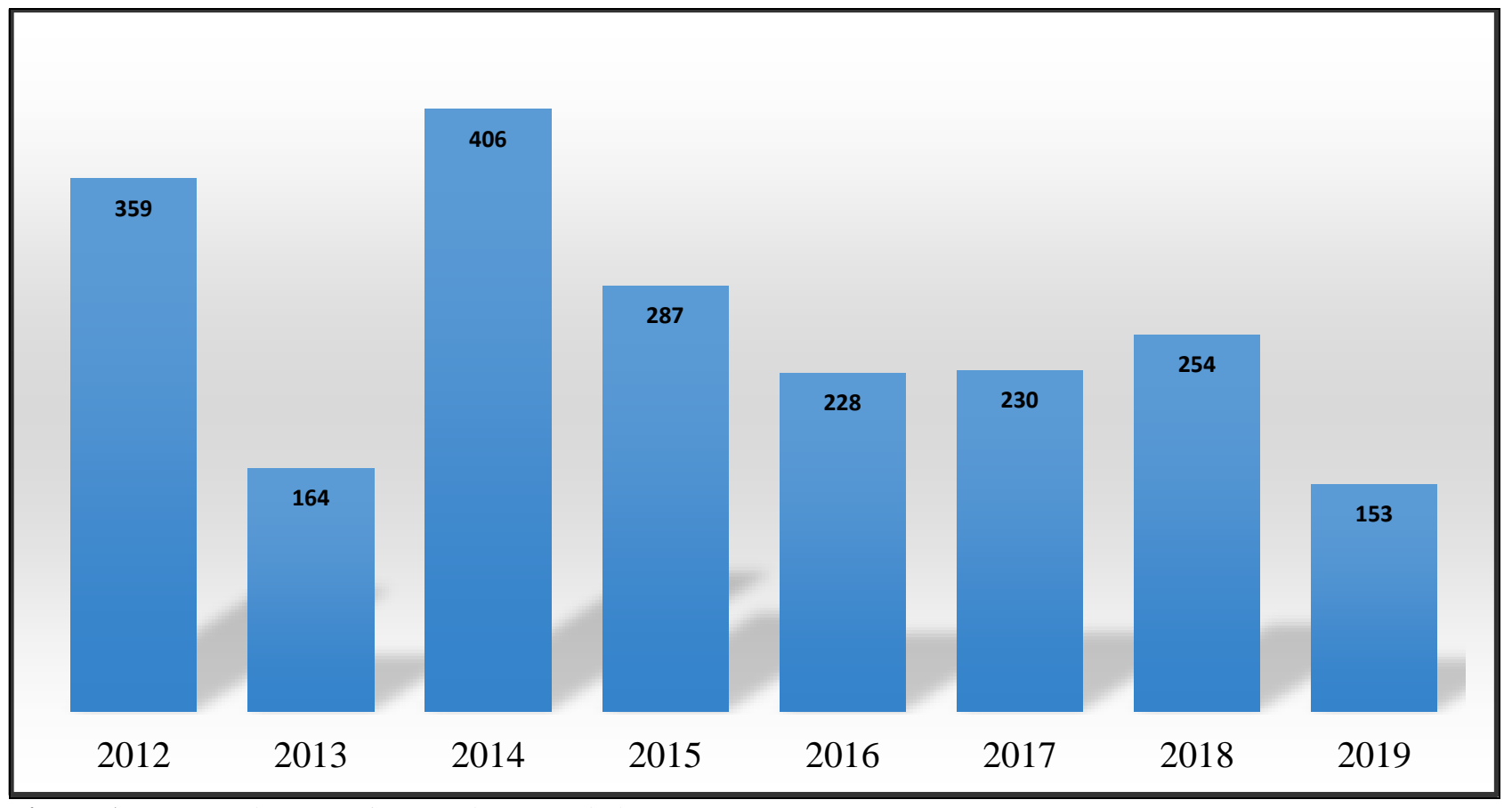

Figura 1. Número de Operaciones Aduaneras de la ARC.

Fuente: elaboración propia basada en los datos estadísticos obtenidos de la Dicomex (2020)

Sumado al número de operaciones aduaneras de la ARC, es importante resaltar que el valor de las mercancías se representa en términos FOB (Free on board), que aplica cuando se cumple la condición de que la empresa vendedora entrega la mercancía a bordo del buque designado por la compradora en el puerto de embarque. Cabe anotar que este valor no incluye seguros ni fletes y que está expresado en dólares americanos (USD). Habría que decir, también, que la conversión del valor de la mercancía de FOB a pesos colombianos se realiza bajo la tasa promedio de cambio del mercado, correspondiente al mes de la operación; no obstante, en este caso se presentarán los datos en USD. De igual modo, el Departamento Administrativo Nacional de Estadística (DANE) valora las operaciones aduaneras en Colombia (Garavito et al., 2014).

Es interesante examinar la Figura 2, donde se observa que durante el período 2012-2019 el valor total FOB de la mercancía importada fue de USD 520658 573, con un valor promedio por año de USD 65082 322, un valor máximo de USD 173832336 en el 2014 y un valor mínimo de USD 13916420 en el 2013. Estos resultados se deben al término de la fase I e inicio de la fase II del programa de modernización de la Fuerza Naval de la ARC, denominado "Orión", bajo los recursos aprobados en el Consejo Nacional de Política Económica y social (CONPES), con número 3460 del 2007, que motivó: la ampliación y modernización de las capacidades aeronavales; la adquisición de unidades de superficie para el patrullaje de la zona económica exclusiva y costera; la actualización, modernización, repotenciación y extensión de la vida de los buques tipo fragatas misileras, y la actualización y el desarrollo de la fuerza submarina de la ARC (Departamento Nacional de Planeación - DNP, 2007). Adicionalmente, se resalta que en la Figura 2 se encuentran incluidas diferentes fuentes de financiación que soportan los programas de mantenimiento de la Fuerza Naval, tales como los contratos con presupuesto nacional y convenios internacionales vigentes con países extranjeros. 


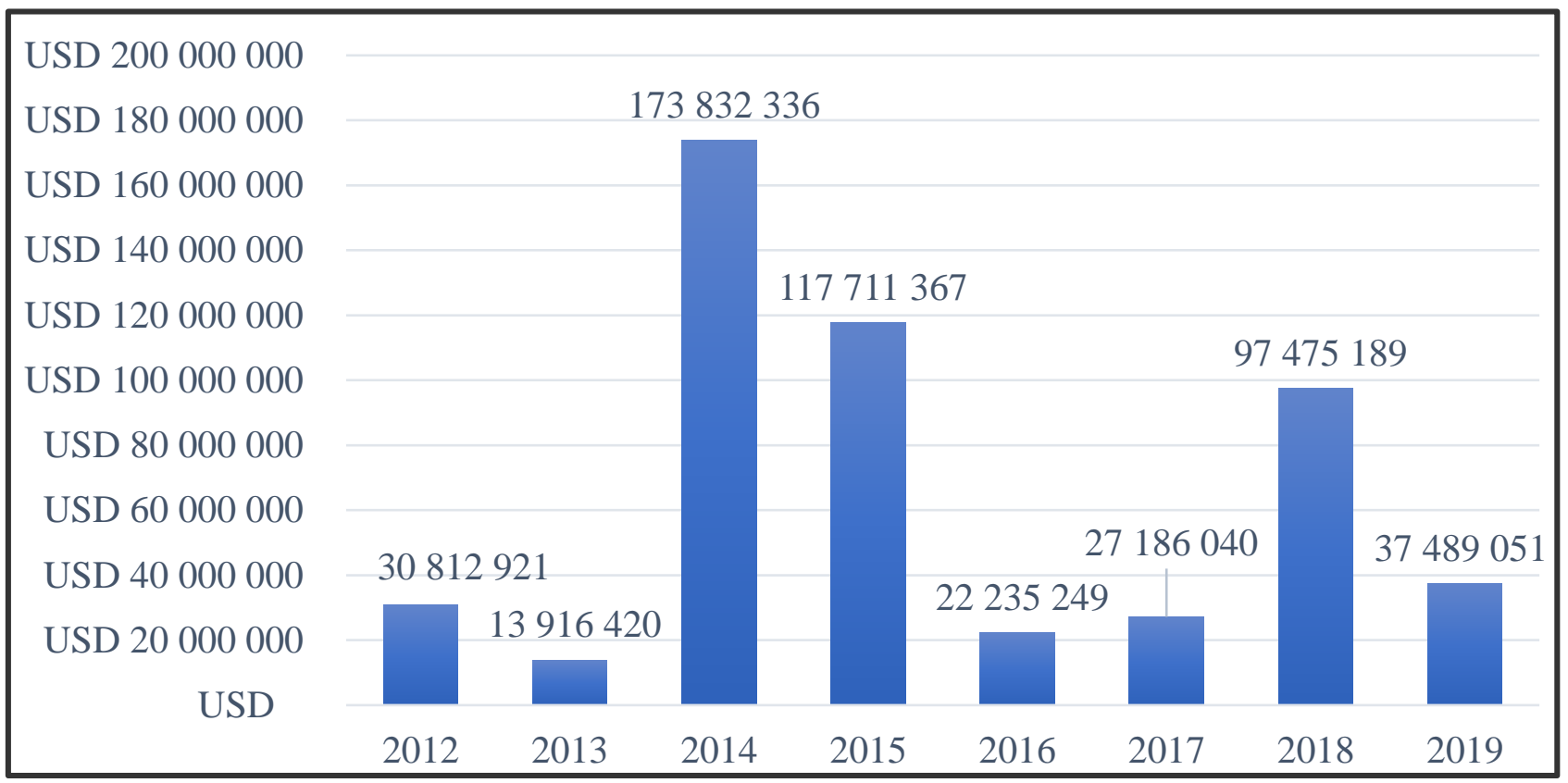

Figura 2. Valor FOB de Importaciones de la ARC 2012 - 2019

Fuente: elaboración propia basada en los datos estadísticos obtenidos de la Dicomex (2020)

De este modo, es oportuno realizar una categorización de las mercancías importadas por la ARC, frente a la fuente de financiación de estos recursos, que permita visualizar el nivel de aporte del presupuesto nacional y las donaciones de los convenios internacionales para soportar la Fuerza Naval (ARC, 2020). Es por ello por lo que en la Figura 3 se puede apreciar, en el período 2012-2019, que el $23 \%$ de las importaciones de mercancías de la ARC corresponde a donaciones de convenios internacionales por valor de USD 121388978 y el $83 \%$ es el resultado de compras con presupuesto nacional por valor de USD 399269 594. Vale la pena recordar que, sin distinción de la fuente de financiación de las mercancías, el cálculo presentado no incluye el valor de seguros ni fletes por tratarse del valor FOB; lo más importante es que la ARC debe prever el pago de aranceles ceñida a la legislación aduanera vigente.

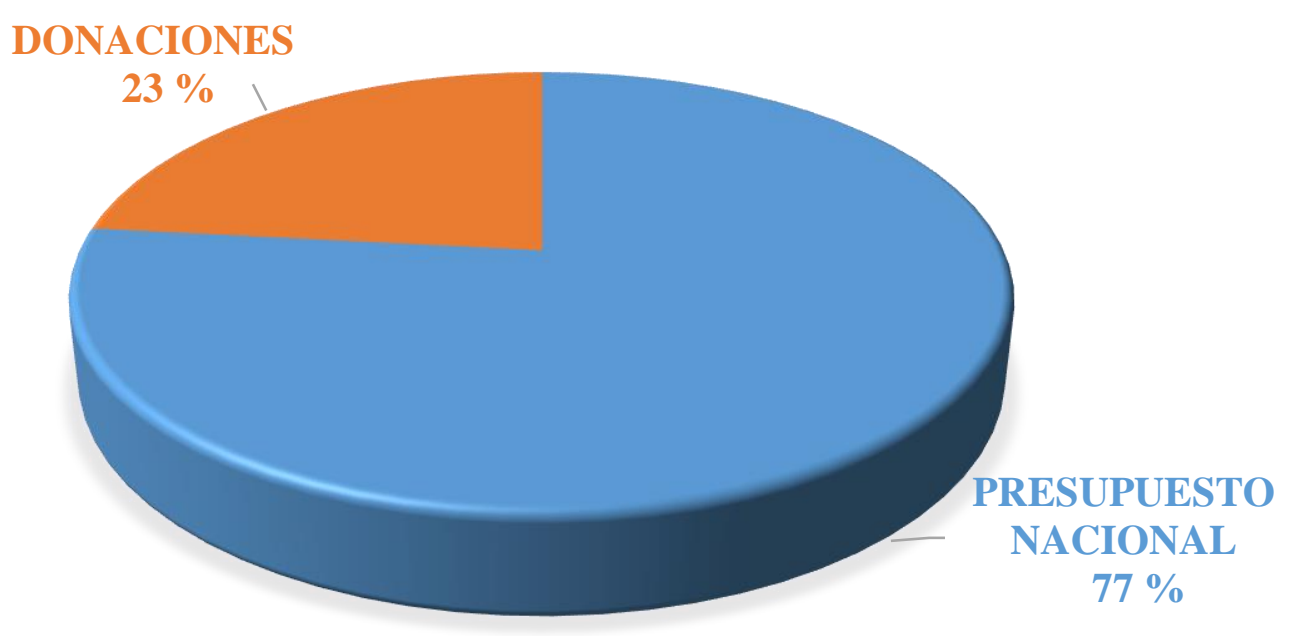

Figura 3. Fuente Recursos en las Importaciones de la ARC.

Fuente: elaboración propia basada en los datos estadísticos obtenidos de la Dicomex (2020) 
Categorización de las operaciones aduaneras de la ARC Las operaciones aduaneras realizadas por la Dicomex se pueden considerar vitales para el sostenimiento del poder naval, puesto que son el soporte de los insumos para el desarrollo de los procesos de mantenimiento de la Fuerza Naval. Esta tarea no ha sido fácil, toda vez que las mercancías de procedencia extranjera de uso recurrente de la ARC están sujetas a partidas arancelarias, gravados con aranceles desde una óptica en derecho aduanero, bajo criterios generales de protección de la industria nacional que no hace ninguna distinción sobre estos bienes considerados para la defensa y seguridad del Estado (Basaldúa, 2014).

Así las cosas, la clasificación de la mercancía importada por la ARC, en el período utilizado en los cálculos anteriores, se ha realizado a través de la nomenclatura del sistema armonizado de designación y codificación de mercancías acogido por Colombia mediante la Ley 646 del 2011 y adoptado en el Arancel de Aduanas vigente mediante el Decreto 2153, del 26 de diciembre 2016, el cual permite presentar la mercancía conforme a la sección y al capítulo correspondiente a la definición de los bienes (MINCIT, 2016).

Con base a la Tabla 1, se pueden distinguir las secciones en las cuales se categorizan las mercancías importadas por la ARC en el período 2012-2019 y se resalta su porcentaje de participación, evidenciada: en la sección XVI, "Máquinas y aparatos, material eléctrico y sus partes", con un 37,16 \%, por valor de USD 193480 157; en la sección XVII, "Material de transporte", con el 32,69 \%, por valor de USD 170222 076, y en la sección XIX, "Armamento y munición", con el 24,08 \%, por valor de USD 125394182. Las secciones citadas cobijan, en su mayoría, los bienes que soportan el mantenimiento de la Fuerza Naval, tales como: buques, submarinos, aeronaves, balsas, vehículos militares, armamento, munición, dotación y equipos que son base de los programas de modernización de los medios navales, decisivos para que el país cuente con un poder naval con gran fuerza disuasiva.

Vale la pena hacer énfasis en la categorización de los bienes de procedencia extranjera de uso recurrente de la ARC, ya que permite profundizar más allá de la clasificación de las mercancías, conforme a las partidas arancelarias consagradas en el Arancel de Aduanas vigente. Esto, toda vez que permite distinguir el impacto que tienen los gravámenes arancelarios en las principales operaciones aduaneras que benefician el soporte de la Fuerza Naval. De esta forma, en el próximo capítulo se desarrollará el análisis correspondiente, partiendo de la información antes presentada.

Tabla 1. Categorización importaciones realizadas por la ARC 2012 - 2019.

\begin{tabular}{|c|l|r|r|r|}
\hline \multicolumn{1}{|c|}{ Categorización } & Clasificación & Valor USD & \% \\
\hline IV & $\begin{array}{l}\text { Productos de las industrias alimentarias; } \\
\text { bebidas, líquidos alcohólicos y vinagre; tabaco } \\
\text { y sucedáneos del tabaco elaborados }\end{array}$ & Raciones de campaña & 71210 & 0,01 \\
\hline V & Productos minerales & Grasa & 79 & 0,00 \\
\hline VI & $\begin{array}{l}\text { Productos de las industrias químicas o de las } \\
\text { industrias conexas }\end{array}$ & Aceites explosivos & 963189 & 0,18 \\
\hline VII & Plástico y caucho con sus manufacturas & Llantas y neumáticos & 1156315 & 0,22 \\
\hline VIII & $\begin{array}{l}\text { Pieles, cueros, peletería y manufacturas de } \\
\text { estas materias }\end{array}$ & Dotación. & 36754 & 0,01 \\
\hline X & $\begin{array}{l}\text { Pasta de madera o de las demás materias } \\
\text { fibrosas } \\
\text { Celulósicas }\end{array}$ & Accesorios, manuales. & 4093204 & 0,79 \\
\hline XI & $\begin{array}{l}\text { Materias textiles y sus manufacturas } \\
\text { XII }\end{array}$ & $\begin{array}{l}\text { Calzado, sombreros y demás tocados, } \\
\text { paraguas, quitasoles, bastones, látigos, fustas, } \\
\text { y sus partes }\end{array}$ & Dotación & 1401073 \\
\hline XIII & $\begin{array}{l}\text { Manufacturas de piedra, yeso, cemento, } \\
\text { amianto (asbesto), mica o materias análogas }\end{array}$ & Dotación & 556787 & 0,68 \\
\hline XV & $\begin{array}{l}\text { Metales comunes y manufacturas de estos } \\
\text { metales }\end{array}$ & $\begin{array}{l}\text { Accesorios, equipos, } \\
\text { herramientas, }\end{array}$ & 502 & 0,27 \\
\hline
\end{tabular}


LA LEGISLACIÓN ADUANERA COLOMBIANA Y SU INFLUENCIA EN EL DESARROLLO DEL PODER NAVAL

\begin{tabular}{|c|c|c|c|c|}
\hline & & repuestos & & \\
\hline XVI & $\begin{array}{l}\text { Máquinas y aparatos, material eléctrico y } \\
\text { sus partes }\end{array}$ & $\begin{array}{c}\text { Accesorios, equipos, } \\
\text { herramientas, } \\
\text { motores, repuestos }\end{array}$ & 193480157 & 37,16 \\
\hline XVII & Material de transporte & $\begin{array}{l}\text { Aeronaves, buques, } \\
\text { botes, balsas, } \\
\text { vehículos militares, } \\
\text { repuestos }\end{array}$ & 170222076 & 32,69 \\
\hline XVIII & Instrumentos y aparatos de óptica & Dotación y equipos & 14114740 & 2,71 \\
\hline XIX & $\begin{array}{l}\text { Armas, municiones, y sus partes y } \\
\text { accesorios }\end{array}$ & $\begin{array}{c}\text { Accesorios, } \\
\text { armamento, equipos, } \\
\text { munición }\end{array}$ & 125394182 & 24,08 \\
\hline $\mathrm{XX}$ & Mercancías y productos diversos & Dotación & 132000 & 0,03 \\
\hline
\end{tabular}

Fuente: elaboración propia basada en los datos estadísticos obtenidos de la Dicomex (2020)

\section{Afectación Arancelaria en las Operaciones Aduaneras de la ARC}

A lo largo de los avances alcanzados en la categorización de las mercancías de uso recurrente por la ARC, se devela que la concentración del $93,93 \%$ de las mercancías importadas por la Fuerza Naval, está enmarcada en las secciones XVI ("Máquinas y aparatos, material eléctrico y sus partes"), XVII ("Material de transporte") y XIX ("Armamento y munición") del Arancel de Aduanas vigente, el cual es el punto de partida para el análisis de la afectación arancelaria a las operaciones aduaneras realizadas en el período de estudio.

De la misma manera, se encuentra en el Arancel de Aduanas la sección XVI, "Máquinas y aparatos, material eléctrico y sus partes", que abarca los capítulos 84 ("Reactores nucleares, calderas, máquinas, aparatos y artefactos mecánicos, partes de estas máquinas y aparatos”) y 85 ("Máquinas y aparatos, material eléctrico y sus partes; aparatos de grabación o reproducción de sonido, aparatos de grabación o reproducción de imagen y sonido en televisión, y las partes y accesorios de estos aparatos"), que corresponden a motores, repuestos, herramientas y accesorios para los buques que forman parte de la capacidad vigente en las Fuerzas Navales, Aviación Naval y botes del Cuerpo de Guardacostas de la Marina Colombiana.

Las mercancías de procedencia extranjera, de las adquisiciones realizadas por la ARC, son categorizadas bajo las partidas arancelarias en los capítulos 84 y 85 (bajo la denominación descrita en la tabla 2); estas partidas se subdividen en 98 subpartidas arancelarias que se encuentran gravadas en su totalidad, de las cuales 92 se encuentran gravadas con el $5 \%, 4$ con el $10 \%$ y 1 con el $15 \%$.

Tabla 2. Clasificación Arancelaria Mercancías Importadas Sección XVI Arancel de Aduanas.

\begin{tabular}{|c|c|c|c|}
\hline Capítulos & Subpartidas & Descripción & $\%$ Arancel \\
\hline $\begin{array}{c}84 \text { "reactores } \\
\text { nucleares, calderas, } \\
\text { máquinas, aparatos } \\
\text { y artefactos } \\
\text { mecánicos, y sus } \\
\text { partes" }\end{array}$ & 48 & 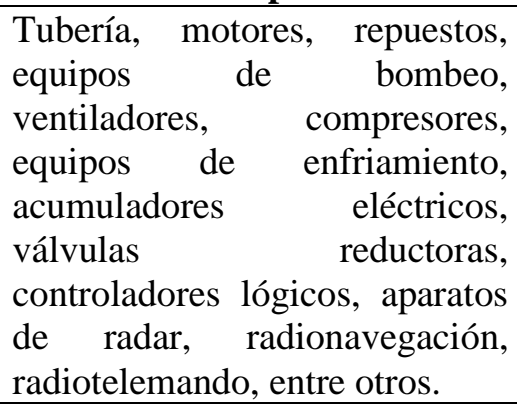 & 5 y 10 \\
\hline $\begin{array}{c}85 \text { "máquinas y } \\
\text { aparatos, material } \\
\text { eléctrico y sus } \\
\text { partes" }\end{array}$ & 50 & $\begin{array}{l}\text { Ups, convertidores estáticos, } \\
\text { baterías, lámparas de seguridad, } \\
\text { generadores de señales, los } \\
\text { demás equipos descritos en el } \\
\text { capítulo } 84 \text { no especificados }\end{array}$ & 5,10 у 15 \\
\hline
\end{tabular}

Fuente: elaboración propia basada en los datos estadísticos obtenidos de la Dicomex ARC (2020 
También cabe revisar que en la sección XVII, "Material de transporte", que abarca los capítulos 86 ("Vehículos y material para vías férreas o similares, y sus partes; aparatos mecánicos [incluso electromecánicos] de señalización para vías de comunicación"), 87 ("Vehículos automóviles, tractores, velocípedos y demás vehículos terrestres, sus partes y accesorios"), 88 ("Aeronaves, vehículos espaciales, y sus partes") y 89 ("Barcos y demás artefactos flotantes"), que corresponden a las aeronaves, buques, botes, balsas, vehículos militares y partes que conforman los principales medios de las Fuerzas Navales, la Aviación Naval y el cuerpo de Infanteria de Marina de la institución.
Las mercancías de procedencia extranjera de las adquisiciones realizadas por la ARC, categorizadas bajo las partidas arancelarias en los capítulos 86, 87, 88 y 89 (ilustrado en la tabla 3), se subdividen en 29 subpartidas arancelarias y se encuentran gravadas en su mayoría, a excepción de la subpartida arancelaria que corresponde a bienes clasificados como helicópteros de peso en vacío superior a $2000 \mathrm{~kg}$, gravada con el $0 \%$. En este sentido, de las 28 subpartidas restantes, 20 están gravadas con el 5 $\%, 2$ con el $10 \%, 4$ con el $15 \%$ y 1 con el $35 \%$, tal y como se ilustra en la tabla 3 .

Tabla 3. Clasificación Arancelaria Mercancías Importadas Sección XVII Arancel de Aduanas.

\begin{tabular}{|c|c|c|c|}
\hline Capítulos & Subpartidas & Descripción & $\%$ Arancel \\
\hline $\begin{array}{c}86 \text { "Vehículos y material } \\
\text { para vías férreas o } \\
\text { similares, y sus partes" }\end{array}$ & 01 & $\begin{array}{l}\text { Vehículos de transporte de } \\
\text { tropa de diferentes tonelajes } \\
\text { con sus respectivos repuestos } \\
\text { y accesorios, incluye } \\
\text { contenedores } \\
\text { almacenamiento. }\end{array}$ & 5 \\
\hline $\begin{array}{l}87 \text { "Vehículos } \\
\text { automóviles, tractores, } \\
\text { velocípedos y demás } \\
\text { vehículos terrestres, sus } \\
\text { partes y accesorios" }\end{array}$ & 12 & $\begin{array}{l}\text { Los demás vehículos de } \\
\text { transporte de tropa de } \\
\text { diferentes tonelajes con sus } \\
\text { respectivos repuestos y } \\
\text { accesorios, que no contemple } \\
\text { el capítulo 86. }\end{array}$ & $5,10,15,35$ \\
\hline \multirow[b]{2}{*}{$\begin{array}{l}88 \text { "Aeronaves, vehículos } \\
\text { espaciales, y sus partes" }\end{array}$} & 01 & $\begin{array}{l}\text { Helicópteros de peso en vacío } \\
\text { superior a } 2.000 \mathrm{~kg} \text {. }\end{array}$ & 0 \\
\hline & 09 & $\begin{array}{l}\text { Los demás aviones, aeronaves } \\
\text { y simuladores de vuelo, de } \\
\text { peso inferior o igual a } 2.000 \mathrm{~kg} \\
\text { con su repuestos y accesorios. }\end{array}$ & 5 \\
\hline $\begin{array}{l}89 \text { "Barcos y demás } \\
\text { artefactos flotantes" }\end{array}$ & 06 & 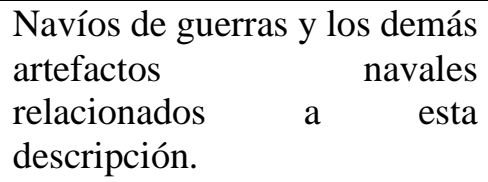 & 5 y 10 \\
\hline
\end{tabular}

Fuente: elaboración propia basada en los datos estadísticos obtenidos de la Dicomex ARC (2020)

De igual manera, se encuentra en la sección XIX, "Armas, municiones, y sus partes y accesorios", que abarca solo el capítulo 93 ("Armas, municiones, y sus partes y accesorios"), que corresponde al armamento, a la munición y a los accesorios para el uso de buques, aeronaves, botes, vehículos militares y personal que tripula los diferentes medios navales y las dependencias de la ARC.

Las mercancías de procedencia extranjera, de las adquisiciones realizadas por la ARC, están categorizadas bajo la partida arancelaria del capítulo 93, el cual se subdivide en 24 subpartidas arancelarias que se encuentran gravadas en su totalidad, de las cuales 23 subpartidas están gravadas con el $15 \%$ y solo una con el $10 \%$.

Una vez realizada una verificación de las partidas arancelarias de las mercancías adquiridas por la ARC de forma recurrente durante los últimos 8 años, queda develado que de 151 subpartidas arancelarias verificadas en los capítulos 84, 85, 86, 87, 88, 89 y 93, que conforman las secciones XVI, XVII y XIX del Arancel de Aduanas colombiano y representan el $93,93 \%$ del valor FOB de las compras de la Fuerza Naval en el período 2012-2019, un $99,9 \%$ está gravado con aranceles, con solo una subpartida 


\section{LA LEGISLACIÓN ADUANERA COLOMBIANA Y SU INFLUENCIA EN EL DESARROLLO DEL PODER NAVAL}

arancelaria exenta de pago. Las demás subpartidas arancelarias tienen obligación de pago por el $5 \%, 10 \%, 15$ $\%$ y $35 \%$; de ahí que se evidencie una afectación presupuestal en el desarrollo de los programas de modernización de la ARC y que, por ende, se limite el fortalecimiento del poder naval del país.

\section{Propuesta de modificación legislativa en pro del mantenimiento del poder naval colombiano}

La ARC ha realizado, en ocasiones anteriores, solicitudes de diferimientos arancelarios para impactar positivamente los programas de modernización de la Fuerza Naval y mantener un poder naval disuasivo ante las amenazas que persisten sobre los intereses nacionales, tales como la situación que persiste en el caribe occidental con Nicaragua y en el caribe oriental con Venezuela.

No obstante, la vigencia de los diferimientos arancelarios es temporal, lo cual no brinda garantías para los futuros proyectos de modernización de la ARC. Así las cosas, es necesario, viable y conducente promover un diferimiento arancelario permanente que contemple las 150 subpartidas arancelarias verificadas en los capítulos 84, 85, 86, 87, 88, 89 y 93, que conforman las secciones XVI, XVII y XIX del Arancel de Aduanas colombiano y representan el 93,93\% del valor FOB de las compras de la Fuerza en el período 2012-2019, brindando un mayor alcance que el conseguido con los decretos 1563 (del 25 septiembre de 2017) y 272 (del 13 de febrero de 2018).

Por lo anterior, se presenta la siguiente propuesta en la Tabla 4, del articulado que podría ser presentado en la sala del Consejo Superior de Política Fiscal (CONFIS) del Ministerio de Hacienda (Minhacienda), con gran potencial de ser avalada por los miembros del comité de Asuntos Aduaneros, Arancelarios y de Comercio Exterior, que sesiona en esa dependencia, con el fin de lograr el respaldo legislativo para los proyectos futuros para el mantenimiento del poder naval de Colombia.

Tabla 4. Propuesta Articulado Proyecto Decreto Diferimiento Arancelario.

\begin{tabular}{|l|l|}
\hline ARTICULADO & \multicolumn{1}{c|}{ CONTENIDO } \\
\hline \multirow{3}{*}{ CONSIDERADO } & $\begin{array}{l}\text { Modificar Decreto 2153 de diciembre del 2016, teniendo en cuenta que el presente } \\
\text { decreto obedece al reconocimiento de los bienes adquiridos por la Armada República } \\
\text { de Colombia en el extranjero para los programas de modernización de la Fuerza Naval } \\
\text { en pro del mantenimiento del Poder Naval colombiano, en este sentido no es coherente } \\
\text { que el Estado siga aplicando aranceles a mencionados bienes por considerarse de Interés } \\
\text { Nacional. Por lo tanto, los aranceles adoptados en el presente decreto entrarán en vigor } \\
\text { a partir de su publicación en el Diario Oficial. Aprobados en la Sesión del Comité de } \\
\text { Asuntos Aduaneros, Arancelarios y de Comercio Exterior, autorizando la reducción del } \\
\text { gravamen arancelario a cero por ciento }(0 \%) \text { para la importación de materias primas y } \\
\text { bienes de capital no producidos en el país. }\end{array}$ \\
\hline
\end{tabular}




\begin{tabular}{|c|c|c|c|c|c|c|}
\hline \multirow{27}{*}{ ARTíCULO 1} & \multicolumn{6}{|c|}{$\begin{array}{l}\text { Establecer un gravamen arancelario del } 0 \% \text { para la importación de los productos } \\
\text { clasificados en las } 151 \text { subpartidas arancelarias relacionadas a continuación: }\end{array}$} \\
\hline & 8407100000 & \begin{tabular}{|l|}
8427200000 \\
\end{tabular} & 8504401000 & 8526920000 & \begin{tabular}{|l|}
8704211000 \\
\end{tabular} & \begin{tabular}{|l|}
8907909000 \\
\end{tabular} \\
\hline & 8407210000 & \begin{tabular}{|l|}
8427900000 \\
\end{tabular} & 8504409000 & 8529101000 & \begin{tabular}{|l|}
8704230000 \\
\end{tabular} & \begin{tabular}{|l|}
301109000 \\
\end{tabular} \\
\hline & 8408100000 & 8431439000 & 8504509000 & 8529109000 & 8704311090 & 9301200000 \\
\hline & 8409100000 & 8451800000 & 8506501000 & 8529909090 & 8704909900 & 9301903000 \\
\hline & 8409999000 & 8467210000 & 8506509000 & 8530809000 & 8708702000 & 9301909000 \\
\hline & 8409999900 & 8467899000 & 8506809000 & 8536101000 & 8708999900 & 9302002100 \\
\hline & 8410900000 & $8471300000 \mid$ & 8507100000 & 8536209000 & 8709110000 & 9302002900 \\
\hline & 8413190000 & 8471410000 & 8507300000 & 8536909000 & 8709190000 & 9303900000 \\
\hline & 8413500000 & 8471490000 & 8507600000 & 8537109000 & 8709900000 & 9304001000 \\
\hline & 8413702900 & \begin{tabular}{|l|l|}
8471800000 \\
\end{tabular} & 8507800000 & 8537200000 & 8710000000 & 9304009000 \\
\hline & 8413919000 & \begin{tabular}{|l|}
8471900000 \\
\end{tabular} & 8507903000 & 8538900000 & 8802120000 & 9305105000 \\
\hline & 8413920000 & \begin{tabular}{|l|}
8473300000 \\
\end{tabular} & 8507909000 & 8539100000 & \begin{tabular}{|l|}
8802209000 \\
\end{tabular} & \begin{tabular}{|l|}
305109000 \\
\end{tabular} \\
\hline & 8414409000 & \begin{tabular}{|l|}
8473500000 \\
\end{tabular} & 8513101000 & 8539299000 & \begin{tabular}{|l|}
8802309000 \\
\end{tabular} & \begin{tabular}{|l|}
9305910000 \\
\end{tabular} \\
\hline & 8414590000 & 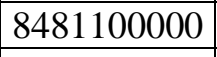 & 8513109000 & 8539490000 & 8803100000 & \begin{tabular}{|l|}
9305911300 \\
\end{tabular} \\
\hline & 8414802100 & 8481300000 & 8517120000 & 8542390000 & 8803200000 & 9305911500 \\
\hline & 8414802290 & \begin{tabular}{|l|}
8481808000 \\
\end{tabular} & 8517180000 & 8543200000 & 8803300000 & 9305911900 \\
\hline & 8414809000 & 8481809900 & 8517700000 & 8543709000 & 8803900000 & 9305919000 \\
\hline & 8414901000 & 8481909000 & 8518300000 & 8544200000 & 8804000000 & 9305990000 \\
\hline & 8415109000 & \begin{tabular}{|l|}
8483109900 \\
\end{tabular} & 8519899000 & 8544429000 & 8805100000 & \begin{tabular}{|l|}
9306210000 \\
\end{tabular} \\
\hline & 8415819000 & \begin{tabular}{|l|}
8483403000 \\
\end{tabular} & 8523492000 & 8544499000 & \begin{tabular}{|l|}
8805210000 \\
\end{tabular} & \begin{tabular}{|l|}
9306291000 \\
\end{tabular} \\
\hline & 8415900000 & 8483409900 & 8525501000 & 8546901000 & 8903991000 & 9306299000 \\
\hline & 8419600000 & \begin{tabular}{|l|}
8487909000 \\
\end{tabular} & 8525601000 & 8546909000 & 8906100000 & \begin{tabular}{|l|}
9306309000 \\
\end{tabular} \\
\hline & 8424100000 & \begin{tabular}{|l|}
8501342000 \\
\end{tabular} & 8525802000 & 8609000000 & \begin{tabular}{|l|}
8906909000 \\
\end{tabular} & \begin{tabular}{|c|}
9306901100 \\
\end{tabular} \\
\hline & 8424909000 & \begin{tabular}{|l|}
8501401900 \\
\end{tabular} & 8526100000 & 8702109000 & \begin{tabular}{|l|}
8907100000 \\
\end{tabular} & \begin{tabular}{|l|}
9306901900 \\
\end{tabular} \\
\hline & 8425499000 & 8501619000 & 8526910000 & 8703100000 & 8907901000 & 9306909000 \\
\hline & 8426411000 & & & & & \\
\hline ARTÍ́CULO 2 & $\begin{array}{l}\text { modifica en } 1 \\
\text { o normas que }\end{array}$ & $\begin{array}{l}\text { Decreto entra } \\
\text { los pertinente } \\
\text { lo modifique }\end{array}$ & $\begin{array}{l}\text { regir a par } \\
\text { artículo } 1^{\circ} \\
\text { aclaren o su }\end{array}$ & $\begin{array}{l}\text { r de su pul } \\
\text { el Decreto } 2 \\
\text { tituyan. }\end{array}$ & ción en e & ario Oficial y \\
\hline
\end{tabular}

Fuente: elaboración propia basada en los datos estadísticos obtenidos de la Dicomex ARC (2020)

\section{DISCUSIÓN}

La Fuerza Naval es una variable vital del poder naval, el cual, como componente militar del poder marítimo, es una de las fuentes del poder nacional y, por ende, una capacidad del Estado que debe mantener una imagen creíble, firme y disuasiva estratégicamente ante las pretensiones permanentes de las amenazas internacionales y en pro de la supervivencia y el futuro de la nación (Hill, 1990).

Por consiguiente, el Estado debe ser coherente con las legislaciones que provee para el mantenimiento de sus capacidades, como es el caso del poder naval, toda vez que no cuenta con un tratamiento favorable para los bienes de procedencia extranjera que son prioritarios para el mantenimiento y la modernización de su Fuerza Naval.
Los bienes requeridos para el sostenimiento de la Fuerza Naval poseen características técnicas especiales cuya producción se le dificulta a la industria nacional y, en su gran mayoría, en la actualidad la industria colombiana no posee empresas nacionales con desarrollos de esta envergadura, tales como: armamento naval, sistemas de armas, artefactos navales, repuestos, equipos, entre otros.

Sin embargo, la legislación aduanera mantiene gravámenes arancelarios en los bienes destinados al mantenimiento del poder naval. A pesar de que en ocasiones han autorizado diferimientos arancelarios temporales gracias a la gestión a contrarreloj de la ARC, persisten en el Arancel de Aduanas las restricciones para las importaciones de dichos bienes, así como se evidenció en las 151 subpartidas arancelarias de las mercancías importadas por la ARC en el período 2012-2019, analizadas en el presente artículo. 
Cabe observar que, de las 151 subpartidas arancelarias analizadas y utilizadas de forma recurrente por la ARC, 79 subpartidas inician su título como "las(os) demás", por no estar descritas en el Arancel de Aduanas, ratificando con esto que los bienes adquiridos por la ARC poseen características especiales no encontradas en la industria nacional ni en la categorización documentada en la normatividad vigente, dificultando las operaciones aduaneras de clasificación y la declaración de las mercancías.

En este orden de ideas, el Estado debe ser coherente con sus fines y velar por el mantenimiento de la Fuerza Naval; en este sentido, debe legislar para que esta dinámica sea menos burocrática, evitando un círculo virtuoso de recaudos y de asignación de recursos entre las mismas instituciones estatales, a pesar de que sean de diferentes ministerios (en este caso, Mindefensa y Minhacienda).

\section{CONCLUSION}

Un acto legislativo con el articulado propuesto anteriormente sería una muestra firme de la voluntad política y estratégica del Estado en apoyo al poder naval colombiano. En el caso de ser aprobado, este daría fin al círculo virtuoso que se presenta en el recaudo de aranceles sobre bienes que forman parte de la ARC y que luego pasan a los ingresos de la nación, compilados por la DIAN y reportados al MINCIT para que, al final, vuelvan a ser asignados en la próxima vigencia fiscal al Mindefensa y, por su conducto, a la Marina de Guerra Colombiana.

Es muy relevante esta iniciativa de reducir los aranceles que persisten en las subpartidas arancelarias de los bienes de procedencia extranjera adquiridos para el mantenimiento de la Fuerza Naval. Sería un gran aporte en la reducción de tiempos en las operaciones aduaneras la disponibilidad de las mercancías y evitar el uso excesivo de las subpartidas catalogadas como "las demás", toda vez que las mercancías importadas por la Armada República de Colombia no se encuentran descritas en el Arancel de Aduanas, recurriendo de esta forma a la clasificación general.

Por último, es importante aclarar que los bienes relacionados en las 151 subpartidas arancelarias, registradas en la propuesta del Decreto con fines de diferimiento arancelario, poseen características técnicas especiales para artefactos navales de procedencia de países como Alemania, Italia, Holanda, Corea del Sur, Bélgica, Francia, España y Estados Unidos de América, los cuales no han sido desarrollados por la industria nacional en la actualidad.

\section{Declaración sobre conflictos de interés}

Las ideas planteadas en este documento, así como las opiniones, comentarios y conclusiones, son responsabilidad del autor de este artículo y son independientes de su institución de afiliación. Así mismo, durante la realización del trabajo y la redacción de este documento no se ha incurrido en ningún conflicto de interés, por tratarse de un documento inédito y original.

\section{REFERENCIAS BIBLIOGRÁFICAS}

Acuña, J. (1994, Octubre). Importancia de la ratificación por Colombia de la Organización Mundial de Comercio. Revista Universidad de los Andes, 28, 1-9 https://revistas.uniandes.edu.co/d oi/abs/10.7440/colombiaint28.19 94.00

Appleyard, D. (2003). Economía internacional. Mexico, Mexico: McGraw Hill. Armada República de Colombia - ARC. (2015). Plan estratégico naval 2015 2018. Bogotá, Colombia: Jefatura de Planeación de la Armada Nacional.

https://www.armada.mil.co/sites/ default/files/plan_estrategico_na val_2016_v2.pdf

Armada República de Colombia - ARC. (2020). Plan estratégico naval 2020 2023. Bogotá, Colombia: Jefatura de Planeación de la Armada Nacional.

https://www.armada.mil.co/sites/ 
default/files/descargas/Plan\%20

Estrategico\%20Naval\%202020-

2023-.pdf

Basaldúa, R. X. (1998). Introducción al derecho aduanero. Buenos Aires, Argentina: Abeledo Perrot.

Carrero, G. P. (2009). Tributación aduanera.

Bogotá, Colombia: Legis.

Castex, R. (1939). Teorías estratégicas. Newport, Estados Unidos de América: Naval War College.

Congreso de la República de Colombia. (2016, 26 de Diciembre) - Ley 2153 de 2016. Arancel de aduanas de Colombia. Diario Oficial $\mathrm{n}^{\circ}$ 50099.

https://normograma.mintic.gov.c o/mintic/docs/decreto_2153_201 $\underline{6 . h t m}$

Chacholiades, M. (1992). Economía internacional. México, México: McGraw-Hill.

Departamento Nacional de Planeación - DNP. (2007, 26 de Febrero). CONPES 3460. Política de consolidación de la seguridad democrática, fortalecimiento de las capacidades del sector defensa y seguridad.

https://colaboracion.dnp.gov.co/ CDT/Conpes/Econ\%C3\%B3mic os/3460.pdf
Dirección de Comercio Exterior de la Armada Nacional - Dicomex (2020). Recopilación archivos estadísticos bases de datos operaciones aduaneras 20122019. Armada República de Colombia.

Dirección de Impuestos y Aduanas Nacionales DIAN. (1996, 29 de Octubre). Resolución $n^{\circ}$ 6592. habilitación deposito aduanero privado a la Armada Nacional.

Dirección de Impuestos y Aduanas Nacionales DIAN. (2005, 09 de Febrero). Resolución $n^{\circ}$ 0970. autorización como usuario aduanero permanente a la Armada Nacional.

Donadio, L. (2013). Controversias en la organización mundial del comercio. Buenos Aires, Argentina: Teseo.

Garavito, A., López, D. y Montes, E. (2014). Índices de valor unitario y quantum del comercio exterior colombiano. Revista Lecturas de Economía, $\quad 80, \quad 9-47$. http://www.scielo.org.co/pdf/le/n 80/n80al.pdf

Hill, R. (1990). Estratégias Marítimas para Potencias Medianas. Buenos Aires, Argentina: Instituto de publicaciones navales. 
LA LEGISLACIÓN ADUANERA COLOMBIANA Y SU INFLUENCIA EN EL DESARROLLO DEL PODER NAVAL

Mahan, A. (2007). La influencia del poder marítimo en la historia. Madrid, España: Ministerio de Defensa.

Ocampo, J. A. y Villar, L. (1992, Junio). Trayectoria y vicisitudes de la apertura económica colombiana. Revista de Economía política pensamiento iberoamerino, 10 , 167-186.

https://core.ac.uk/download/pdf/ 148763267.pdf\#page $=167$

Queralt, J. M. (2007). Manual de Derecho Tributario. Pamplona, España: Aranzadi.

Rivera, M. (1993, Abril). Del sobreproteccionismo a la apertura comercial. Revista política y cultura, 2, 137 - 163. https://doctrina.vlex.com.mx/vid/ sobreproteccionismo-aperturacomercial-204664129

Steinberg, F. (2004). La nueva teoría del comercio internacional y la política comercial estratégica. Madrid, España: Universidad Autónoma de Madrid.

Till, G. (2007). El poder maritimo. Buenos Aires, Argentina: Instituto de publicaciones navales.

Valdivia, A. E. (2010). El sistema tributario. Revista Academica de Economía, 140. http://www.eumed.net/cursecon/ecolat/c $\underline{\mathrm{u} / 2010 / \mathrm{aevb} 2 . \mathrm{htm}}$ 